\title{
LXXVIII. Some observations on the electrolysis of salts
}

\section{Robert Hare M.D.}

To cite this article: Robert Hare M.D. (1843) LXXVIII. Some observations on the electrolysis of salts, Philosophical Magazine Series 3, 22:147, 461-467, DOI: $10.1080 / 14786444308636420$

To link to this article: http://dx.doi.org/10.1080/14786444308636420

曲 Published online: 01 Jun 2009.

Submit your article to this journal \lceil

Џll Article views: 2

Q View related articles $\sqsubset$ 
Dr. Hare and Prof. Daniell on the Electrolysis of Salts. 4.61

large vacuities must have been left in others. Had such convulsions ever taken place, traces of them must have been conspicuous; but, in fact, nothing of the kind has been detected, even in a single instance.

If, however, the movements be supposed to have taken place before the rocks had become perfectly solid, in fact, whilst they were in a state which permitted segregatory or molecular action, the question will no longer be one of simple mechanical displacement, but rather concern those forces which determine the movements of minute particles of matter.

Whether any actions thus arising could have given birth to the diversified phænomena we have considered seems to have no practical bearing, and therefore forms no part of this inquiry.

4 Clarence-street, Penzance, 1842, Sept. 29th.

LXXVIII. Some Observations on the Electrolysis of Salts. By Robert Hare, M.D., Prof. of Chem. Univ. of Pennsylvania: with a Reply thereto by J. Frederic Daniell, For. Sec. R.S., Prof. of Chem. King's College, London; in a Letter addressed to R. Phillips, Esq., F.R.S., \&c.

My dear Sir,

MY friend Dr. Hare has done me the favour to send me a copy of a paper which he has recently published, entitled "An Effort to refute the arguments advanced in favour of the existence, in the Amphide Salts, of Radicals consisting, like cyanogen, of more than one element." Amongst these arguments, with the majority of which I am not disposed at present to interfere, he ranks the deductions which $\mathrm{I}$ have drawn from my electrolytic experiments. To observations made by such high authority I am desirous of making some reply, and as the subject is of considerable importance, I am induced to think that you will endeavour to make room in the Philosophical Magazine both for so much of the paper as concerns the electrolysis, and the few brief remarks upon it which I wish to make.

Dr. Hare observes, -

" 66. The last argument in favour of the existence of salt radicals, which I have to answer, is that founded on certain results of the electrolysis of saline solutions.

" 67 . On subjecting a solution of sulphate of soda to electrolysis, so as to be exposed to the current employed, simultaneously with some water in a voltameter, Daniell alleges that, for each equivalent of the gaseous elements of water evolved in the voltameter, there was evolved at the cathode and anode, not only a like quantity of those elements, but likewise an equal number of equivalents of soda 


\section{Dr. Hare's Observations on the Electrolysis of Salts,}

and sulphuric acid. This he considers as involving the necessity, agreeably to the old doctrine, of the simultaneous decomposition of two electrolytic atoms in the solution, for one in the voltameter; while, if the solution be considered as holding oxysulphionide of sodium, instead of sulphate of soda, the result may be explained consistently with the law ascertained by Faraday. In that case, oxysulphion would be carried to the anode, where, combining with hydrogen, it would cause oxygen to be extricated, while sodium, carried to the cathode, and deoxidizing water, would cause the extrication of hydrogen.

“ 68. Dr. Kane, alluding to the experiments above mentioned, and some others which I shall mention, alleges that 'Professor Daniell considers the binary theory of salts to be fully established by them.'

"69. Notwithstanding the deference which I have for the distinguished inventor of the constant battery, and disinclination for the unpleasant task of striving to prove a friend to be in the wrong, being of opinion that these inferences are erroneous, $I$ feel it to be my duty as a teacher of the science, to show that they are fourded upon a misinterpretation of the facts appealed to for their justification.

“ 70. It appears to me, that the simultaneous appearance of the elements of water, and of acid and alkali, at the electrodes, as above stated, may be accounted for, simply by that electrolyzation of the soda, which must be the natural consequence of the exposure of the sulphate of that base in the circuit. I will, in support of the exposition which I am about to make, quote the language of Professor Daniell, in his late work, entitled 'Introduction to Chemical Philosophy,' page 413 :-

"Thus we may conceive that the force of affinity receives an impulse which enables the hydrogen of the first particle of water, which undergoes decomposition, to combine momentarily with the oxygen of the next particle in succession; the hydrogen of this again, with the oxygen of the next; and so on till the last particle of hydrogen communicates its impulse to the platinum, and escapes in its own elastic form.'

“71. The process here represented as taking place in the instance of the oxide of hydrogen, takes place, of course, in that of any other electrolyte.

"72. It is well known, that when a fixed alkaline solution is subjected to the voltaic current, the alkali, whether soda or potassa, is decomposed; so that if mercury be used for the cathode, the nascent metal, being protected by uniting therewith, an amalgam is formed. If the cathode be of platinum, the metal, being unprotected, is, by decomposing water, reconverted into an oxide as soon as evolved. This shows, that when a salt of potassa or soda is subjected to the voltaic current, it is the alkali which is the primary object of attack, the decomposition of the water being a secondary result.

"73. If in a row of the atoms of soda, extending from one electrode to the other, while forming the base of a sulphate, a series of electrolytic decompositions be induced from the cathode on the right, 
to the anode on the left, by which each atom of sodium in the row will be transferred from the atom of acid with which it was previously combined, to that next upon the right, causing an atom of the metal to be liberated at the cathode; this atom, deoxidizing water, will account for the soda and hydrogen at the cathode. Meanwhile the atom of sulphate on the left, which has been deprived of its sodium, must simultaneously have yielded to the anode the oxygen by which this metal was oxidized. Of course the acid is left in the hydrous state, usually called free, though more correctly esteemed to be that of a sulphate of water.

" 74. I cannot conceive how any other result could be expected from the electrolysis of the base of sulphate of soda, than that which is here described.

"75. I will, in the next place, consider the phænomena observed by Prof. Daniell, when solutions of potassa and sulphate of copper, separated by a membrane, were made the medium of a voltaic current.

" 76. Of these I here quote his own account, Philosophical Magazine and Journal, vol. xvii. p. $172^{*}$.

“77. It will be admitted, that agreeably to the admirable researches of Faraday, there are two modes in which a voltaic current may be transmitted, conduction and electrolyzation. In order that it may pass by the last-mentioned process, there must be a row of anions and cathions forming a series of electrolytic atoms extending from the cathode to the anode. It is not necessary that these atoms should belong to the same fluid. A succession of atoms, whether homogeneous, or of two kinds, will answer, provided either be susceptible of electrolyzation. Both of the liquids resorted to by Daniell, contained atoms susceptible of being electrolyzed. If his idea of the composition of sulphate of copper, and the part performed by the potassa, were admitted for the purpose of illustration, we should, on one side of the membrane, have a row of atoms consisting of oxysulphion and copper; on the other, of oxygen and hydrogen.

“78. Recurring to Daniell's own description of the electrolyzing process, above quoted, an atom of copper near the anode being liberated from its anion, oxysulphion, and charged with electricity, seizes the next atom of oxysulphion, displacing and charging an atom of copper therewith united. The cupreous atom thus charged and displaced, seizes a third atom of oxysulphion, subjecting the copper, united with it, to the same treatment as it had itself previously met with. This process being repeated by a succession of similar decompositions and recompositions, an electrified atom of copper is evolved at the membrane, where there is no atom of oxysulphion. Were there no other anion to receive the copper, evidently the electrolyzation would not have taken place; but oxygen, on the one side of the membrane, must succeed to the office performed by oxysulphion on the other side; while hydrogen, in like manner, must succeed to the office of the copper.

"79. Such being the inevitable conditions of the process, how

* This account, having already appeared in this Journal, it is not necessary to repeat. 


\section{Dr. Hare's Observations on the Electrolysis of Salts,}

can it be correctly alleged by Professor Daniell, the transfer of the copper being arrested at the membrane, that as this metal ' can find nothing to combine with,' it gives up its electrical charge to the hydrogen, which proceeds to the cathode? As hydrogen cannot be present, excepting as an ingredient in water, how can it be said that the copper can discharge itself upon the hydrogen, without combining with the oxygen necessarily liberated at the same time by the electrolytic process? How could the copper, in discharging itself to a cathion, escape a simultaneous seizure by an anion? Would not the oxidizement of this metal be a step indispensable to the propagation of that electrolytic process, by which alone the hydrogen could, as alleged, 'pass to the platinode,' i. e. cathode?

“ 80 . In these strictures $I$ am fully justified by the following allegations of Faraday, which I quote from his Researches, 826, 828 :-

" "A single ion, i. e. one not in combination with another, will have no tendency to pass to either of the electrodes, and will be perfectly indifferent to the passing current, unless it be itself a compound of more elementary ions, and so subject to actual decomposition.

" 'If, therefore, an ion pass towards one of the electrodes, another ion must also be passing simultaneously to the other electrode, although, from secondary action, it may not make its appearance.'

“ $8 \mathrm{I}$. In explanation of the mixed precipitates produced upon the membrane, I suggest that the hydrated oxide resulted from chemical reaction between the alkali and acid, the oxide from the oxygen of the water or potassa acting as a cathion in place of that of the oxide of copper; also that the metallic copper is to be attributed to the solutions acting both as conductors and as electrolytes; so that, at the membrane, two feeble electrodes were formed, which enabled a portion of the copper to be discharged without combining with an anion, and a portion of oxygen to be discharged without uniting with a cathion. In this explanation I am supported by the author's account of a well-known experiment by Faraday, in which a solution of magnesia and water was made to act as electrodes at their surfaces respectively.

" 82. There can, I think, be no better proof that no reliance should be placed on the experiments with membranes, in this and other cases where the existence of compound radicals in acids is to be tested, than the error into which an investigator, so sagacious as $\mathrm{my}$ friend Professor Daniell, has been led, in explaining the complicated results.

" 83. The association of two electrolytes, and the chemical reaction between the potassa and acid, which is admitted to have evolved the hydrated oxide, seem rather to have created difficulties than to have removed them.

" 84. In this view of the subject I am supported by the opinion of Faraday, as expressed in the following language :-

" . When other metallic solutions are used, containing, for instance, peroxides, as that of copper combined with this or any decomposable acid, still more complicated results will be obtained, which, 
viewed as the direct results of electro-chemical action, will, in their proportions, present nothing but confusion; but will appear perfectly harmonious and simple, if they be considered as secondary results, and will accord in their proportions with the oxygen and hydrogen evolved from water by the action of a definite quantity of electricity.'

“ 85. I cannot conceive that in any point of view the complicated and 'confused' results of the experiment of Daniell with electrolytes separated by membranes, are rendered more intelligible by supposing the existence of salt radicals. I cannot perceive that the idea that the anion in the sulphate is oxysulphion, makes the explanation more satisfactory than if we suppose it to be oxygen. Were a solution of copper subjected to electrolysis alone, if the oxide of copper were the primary object of the current, the result would be analogous to the case of sodium, excepting that the metal evolved at the cathode, not decomposing water, would appear in metallic form. If water be the primary object of attack, the evolution of copper would be a secondary effect.

" 86. It is remarkable, that after I had written the preceding interpretation of Daniell's experiments, I met with the following deductions stated by Matteuchi as the resuit of an arduous series of experiments, without any reference to those of Daniell above mentioned. It will be perceived that these deductions coincide perfectly with mine.

“ 87. I subjoin a literal translation of the language of Matteuchi from the Annales de Chimie et de Physique, tom. Ixxiv. 1840, p. 110 :-

" When salt, dissolved in water, is decomposed by the voltaic current, if the action of the current be confined to the salt, for each equivalent of water decomposed in the voltameter, there will be an equivalent of metal at the negative pole, and an equivalent of acid, plus an equivalent of oxygen, at the positive pole. The metal separated at the negative pole will be in the metallic state, or oxidized according to its nature. If oxidized, an equivalent of hydrogen will be simultaneously disengaged by the chemical decomposition of water.'

" 88. Thus it seems that the appearance of acid and oxygen at the anode, and of alkali and hydrogen at the cathode, which has been considered as requiring the simultaneous decomposition of two electrolytes upon the heretofore received theory of salts, has, by Matteuchi, been found to be a result requiring the electrolysis of the metallic base only, and consequently to be perfectly reconcilable with that theory.

“ 89. In fact I had, from the study of Faraday's Researches, taken up the impression, that the separate appearance of an acid and base previously forming a salt, at the voltaic electrodes, was to be viewed as a secondary effect of the decomposition of the water or the base; so that acids and bases were never the direct objects of electrolytic transfer."

In the first place I must thank Dr. Hare for the perfect courtesy with which he has striven "to prove a friend to be in the wrong," and giving him credit for feeling, as I do, that Phil. Mag. S. 3. Vol. 22. No. 147. June 1843. 2 I 


\section{Dr. Hare and Prof. Daniell on the Electrolysis of Salts}

I had much rather be so proved to be in the wrong than that any error which I may have cornmitted should remain uncorrected, I shall proceed to prove that, owing to his overlooking one important result of the electrolysis, he is not in the right.

It appears, then, to Dr. Hare, that in the electrolysis of saline solutions, the simultaneous appearance of the elements of water, and of acid and alkali, at the electrodes may be accounted for simply by that electrolyzation of the soda (taking sulphate of soda as an illustration) which must be the natural consequence of the exposure of the sulphate of that base in the circuit. I presume that by the term natural consequence is meant that such a result might have been, à priori, expected: but there is nothing, I think, that I should have anticipated less than that a solution of sulphate of soda would be affected by the voltaic current precisely in the same way as a solution of caustic soda, or that the powerful affinities of the acid and the base should have no influence upon the result.

The whole of Dr. Hare's argument is founded upon the supposition that both in an aqueous solution of soda and of sulphate of soda, "the alkali is the primary object of attack, the decomposition of the water being a secondary result." But he has neglected the important fact, that in neither case is it the sodium and the oxygen which alone travel in the circuit; but in the first case the water, and in the second case the acid accompanies the oxygen to the anode. In my experiments with the diaphragm cells, by which the liquid products of the electrolysis may be kept sufficiently separate, I have proved this over and over again. Indeed I should bave joined most heartily in the wonder of my friend at the failure of my own "sagacity," if I had not established this point beyond the possibility of a doubt. It will be easy, I am sure, for Dr. Hare to convince himself by a few easy experiments that a solution of sulphate of soda submitted to electrolysis becomes acid in the zincode division of a diaphragm cell, not only by the abstraction of sodium from it, but by the accumulation of acid transferred from the platinode division; just as he will find an accumulation of soda in the latter arising from the secondary action of the sodium transferred from the former.

The same oversight of the fact, that in these processes of the electrolysis of secondary compounds the acid necessarily accompanies the oxygen to the zincode while the metal travels to the platinode, has misled Dr. Hare in his consideration of the phænomena observed by me when solutions of potassa and sulphate of copper, separated by a membrane, were made the medium of a voltaic circuit. It is this oversight alone which 
has rendered them to him complicated and confused. To account for the deposition of the metal upon the diaphragm, he is reduced to the necessity of supposing that " the metallic copper is to be attributed to the solutions acting (in some manner to me unintelligible) both as conductors and electrolytes."

According to my explanation the copper is arrested on its passage to the platinode by the impossibility of its combining even temporarily with the hydrate of potassa. In its state of oxide it is also arrested on account of its being insoluble. The metal, therefore, yields its charge to the hydrogen of the hydrate which is evolved in its place at the platinode. Both the copper of the sulphate and the oxygen of the hydrate are evolved upon the membrane and enter into secondary union, if there be time for the completion of the combination; but this secondary combination is not necessary to the electrolysis, and if the process be very rapid does not completely take place.
I remain, my dear Sir, very faithfully yours,
King's College, London,
J. F. Daniell. May $11,1843$.

\section{On Rthogen and AEthonides. By WILliaM H, BALMAIN*.}

$A^{\mathrm{T}}$ $T$ the commencement of the present year I made some experiments, with the hope of obtaining compounds of boron and silicon with nitrogen; and was successful so far as to obtain compounds consisting of boron and nitrogen together with certain metals, which are possessed of some very remarkable properties. The results of these experiments, with some remarks upon their bearing upon the science of chemistry, and a few facts proving the existence of analogous compounds of silicon and nitrogen, were published in the Phil. Mag. for October, 1842. Since that time I have succeeded in isolating the compound of nitrogen and boron, and have given it the name of Ethogen, from ai $\theta \omega v$ and yeivopal, because it produces, by uniting with the metals, compounds which glow with a peculiarly beautiful phosphorescent light when heated before the blowpipe in the oxidizing flame. And I think its compounds may with propriety be named Fthonides.

Preparation of AEthogen.-Heat to redness seven parts of finely powdered anhydrous boracic acid with nine parts of melon, in a crucible lined with charcoal; and immediately the

* Communicated by the Chemical Society; having been read December 6,1842 . 